\title{
Edulkation und Graded Exposure vs. Bewegungsangst
}

\author{
Ralf Schesser
}

Trotz eines fundierten Clinical Reasonings stellt sich kein Therapierfolg ein? Die Schuld wird nicht selten beim „schwierigen“ Patienten gesucht. Erst im Behandlungsverlauf kann deutlich werden, dass maladaptive Kognitionen zu Ängsten führen und der Genesung im Wege stehen. Ein Fallbeispiel zeigt, wie Kinesiophobie zur Barriere in der Therapie wird. Um diese zu überwinden, kann die Kombination aus Edukation und Expositionstherapie helfen.

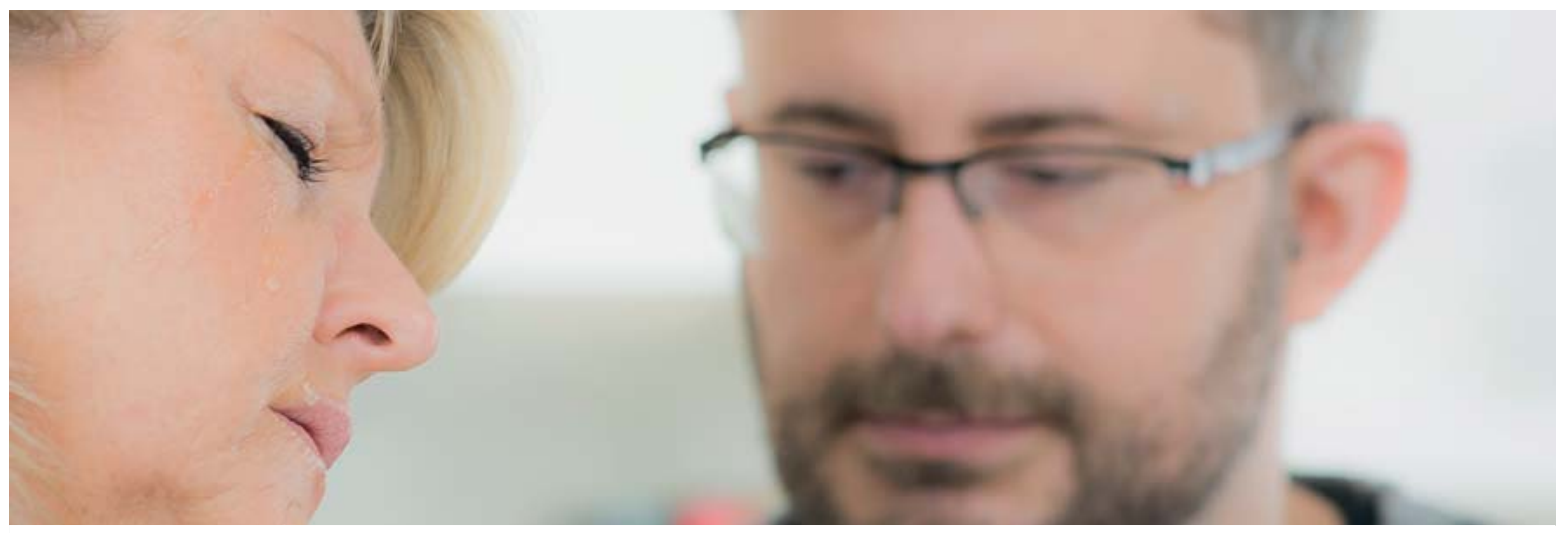

Quelle: Racle Fotodesign/stock.adobe.com (Symbolbild).

Sabine F. ist Lageristin. Beim Heben eines schweren Pakets verspürt die 32-Jährige einen stechenden Schmerz im Rücken, kann sich anschließend kaum noch aufrichten und bricht ihre Arbeit ab. Drei Stunden später imponieren rechtsseitig Schmerzen im Bein sowie eine Taubheit im Fuß. Am nächsten Tag konsultiert sie ihren Hausarzt. Dieser attestiert ihr die Arbeitsunfähigkeit, empfiehlt eine mehrtägige Bettruhe und verordnet ihr ein nichtsteroidales Antirheumatikum (NSAR).

Die sportliche Patientin liebt Klettern, Wandern und Skifahren. Sie hatte zuvor weder Rückenschmerzen noch Beinprobleme. Ihr Allgemeinzustand ist sehr gut, es liegen keine schwerwiegenden Pathologien oder relevanten Operationen vor.
BSV L5/S1 Weil sich die Beschwerden nach einer Woche nicht bessern, sucht Frau F. einen Orthopäden auf. Die MRT-Bildgebung zeigt einen rechtsseitigen Bandscheibenvorfall im Segment L5/S1, der die Nervenwurzel zwar grenzwertig, aber ohne OP-Indikation komprimiert. Eine Cauda-Equina-Symptomatik oder Rückenmarkskompressionszeichen lagen nicht vor.

In der Folge wurde die 32-Jährige in der orthopädischen Praxis eine Woche lang täglich mit Elektrotherapie behandelt. Weil auch diese Intervention die Schmerzsymptomatik nicht linderte, erhielt Sabine F. eine Erstverordnung für Physiotherapie mit der Diagnose „Lumboischialgie rechts bei Bandscheibenvorfall (BSV) L5/S1“. Seit dem Arbeitsunfall waren mittlerweile fast drei Wochen vergangen. 


\section{Assessment}

\section{Subjektive Befunderhebung}

Hauptproblem Beim Erstkontakt gab Frau. Fals Hauptproblem an, dass sie v. a. beim Bücken schmerzbedingt bewegungseingeschränkt sei. Zudem fühle sich ihr rechtes Bein „sehr komisch“ an und sei „nicht mehr so kraftvoll“. Sie war frustriert, weil sie weder ihrer Arbeit, noch ihren sportlichen Ambitionen nachgehen konnte.

\section{Schmerzanamnese}

Die in der unteren LWS lokalisierten Schmerzen beschrieb sie als „ziehend“, „drückend“ und „dumpf in der Tiefe“ sitzend. Die Schmerzen träten intermittierend auf und erreichten einen Maximalwert von 6/10 auf der Numerischen Ratingskala (NRS).

Die Schmerzen im rechten Gesäß seien „ziehend“, teilweise „brennend“ oder „elektrisierend“. Sie zögen dorsolateral in das Bein bis zur Kleinzehe. Wenn der Rückenschmerz stärker wurde, erreichte der Gesäßschmerz einen Wert von bis zu 8/10 NRS. Überdies bestünde von Beginn an ein permanentes Taubheitsgefühl am Außenrand des rechten Fußes ( $\bullet$ Abb. $\mathbf{1})$.

Als schmerzverstärkende Aktivitäten gab die Patientin das Bücken, Sockenanziehen, Heben sowie ein mehr als 20-minüiges Sitzen und auch das Aufstehen an. Schmerzlindernd wirkten dagegen Gehen, sanfte Bewegungen, Stufenlagerung und Liegen. Wärme und die vom Hausarzt verordnete Medikation mit NSAR würden ihre Schmerzsensationen ebenfalls mindern.

Ferner bemerkte sie, dass sich ihr Rücken morgens „steif“ anfühle und schmerzhaft sei. Im Tagesverlauf nähme diese Problematik je nach Belastungssituation zu. In der Nacht wache Frau F. aufgrund der Rücken- und Beinschmerzen öfters auf und könne erst nach Bewegung und Lagewechsel wieder einschlafen.

\section{Psycho-Soziale Faktoren}

Die Patientin erzählte von ihrem sicheren Job mit gutem Arbeitsklima und netten Kollegen. Ihre Arbeit als Lageristin bereite ihr trotz der großen körperlichen Anstrengung beim Heben und Tragen schwerer Lasten viel Freude. Privat lebe sie in einer glücklichen Beziehung zusammen mit ihrem Ehemann.

Angelpunkt Eher beiläufig berichtete Frau F., ein wenig Angst vor einer Verstärkung der Schmerzen durch Aktivität zu haben und sich durch Bewegung und Belastung weitere Schäden zuzufügen. Angesichts dessen schonte sie sich und mied jegliche schmerzauslösende Aktivität. Dabei äußerte sie erste Bedenken, ihre Sportarten wieder ausüben zu können.

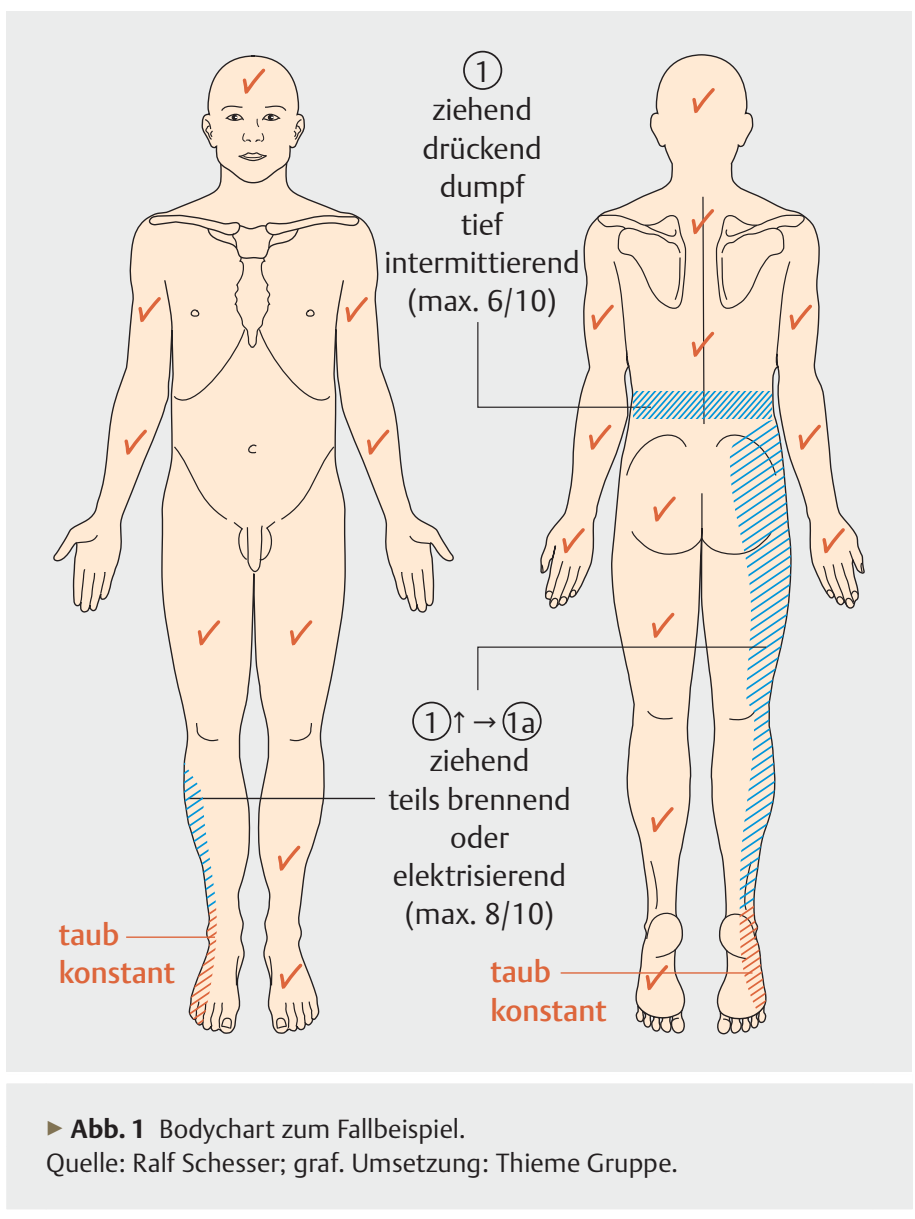

\section{Objektive Befunderhebung}

Bei der Analyse der Statik zeigte sich ein deutlicher Shift des Oberkörpers nach links. Bei Flexion der LWS erreichte die Patientin einen Finger-Boden-Abstand von lediglich $50 \mathrm{~cm}$, bevor die ziehenden Beinschmerzen auftraten. Bei 20\%iger LWS-Extension oder 50\%iger rechtsseitiger Lateralflexion klagte sie über ihre zentral lokalisierten Rücken- sowie den ausstrahlenden Beinschmerz. Die HWS-Flexion verstärkte, deren Extension linderte den Schmerz.

Neurologie Bei der neurologischen Untersuchung war der Achilles-Sehnen-Reflex rechts leicht abgeschwächt. Der wiederholte Zehenstand war rechts schneller ermüdbar und zudem wackelig. Gleichzeitig imponierte eine ausgeprägte Hyposensibilität am rechten Fußaußenrand. Der Straight-Leg-Raise (SLR) löste rechtsseitig bei $30^{\circ}$ den Beinschmerz aus und steigerte sich bei Dorsalextension im Oberen Sprunggelenk. Der linksseitige SLR bewirkte bei $70^{\circ}$ ein Ziehen im dorsalen Oberschenkel. 
- Tab. 1 Hypothesenkategorien des Clinical Reasoning anhand des Fallbeispiels [3].

\begin{tabular}{|c|c|c|}
\hline Kategorien & Unterpunkte & Ersthypothese \\
\hline $\begin{array}{l}\text { Funktionsstörung/ } \\
\text { Dysfunktion }\end{array}$ & $\begin{array}{l}\text { Beschreibung der Funktionsstörung anhand der ICF-Ebenen: } \\
\text { - Körperfunktionen und -strukturen } \\
\text { - Aktivitäten } \\
\text { - Partizipation }\end{array}$ & $\begin{array}{l}\text { - Schmerz und Bewegungseinschränkung in Rücken } \\
\text { und Bein beim Sitzen und Beugen } \\
\text { - Arbeiten und Sport nicht möglich } \\
\text { - Frust und Bedenken aufgrund dieses Zustandes }\end{array}$ \\
\hline $\begin{array}{l}\text { pathobiologische } \\
\text { Mechanismen }\end{array}$ & $\begin{array}{l}\text { Gewebemechanismen: } \\
\text { - Wundheilungsphase } \\
\text { - Gewebestabilität } \\
\text { Schmerzmechanismen anhand des, Mature Organism Model‘ (MOM) [4]: } \\
\text { - Input: periphere Mechanismen } \\
\text { - nozizeptiv: } \\
\text { • Entzündung } \\
\text { - Ischämie } \\
\text { • Mechanik } \\
\text { - Processing: } \\
\text { - zentral neuropathisch } \\
\text { - noziplastisch } \\
\text { - kognitiv-affektiv } \\
\text { - Output: } \\
\text { - motorische Funktionsstörungen } \\
\text { - Dysfunktion von: } \\
\text { • endokrinem System } \\
\text { - hormonellen System } \\
\text { • Vegetativum } \\
\text { • Immunsystem }\end{array}$ & $\begin{array}{l}\text { Gewebemechanismen: } \\
\text { - Gewebeschaden kurz nach der Proliferationsphase } \\
\text { Schmerzmechanismen: } \\
\text { - Input: } 60 \% \\
\text { - } 25 \% \text { nozizeptiv } \\
\text { - } 35 \% \text { peripher neuropathisch } \\
\text { - Processing: } 30 \% \\
\text { - Output: } 10 \%\end{array}$ \\
\hline $\begin{array}{l}\text { Quellen der } \\
\text { Symptome }\end{array}$ & Auflistung der wahrscheinlichsten Schmerzgeneratoren und -strukturen & $\begin{array}{l}\text { - LWS (L4/L5/S1) } \\
\text { - Bandscheiben } \\
\text { " Nervenwurzel L5 und/oder S1 }\end{array}$ \\
\hline $\begin{array}{l}\text { Vorsichtsmaß- } \\
\text { nahmen und } \\
\text { Kontraindikationen }\end{array}$ & $\begin{array}{l}\text {,Red Flags' als absolute Kontraindikation } \\
\text { Relative Vorsichtsmaßnahmen: } \\
\text { " schlechter Allgemeinzustand } \\
\text { " diverse Grunderkrankungen (Rheuma, Diabetes etc.) } \\
\text { " progrediente Nervenwurzelprobleme } \\
\text { " frische Operationen } \\
\text { " sich verschlechternde Zustände oder Pathologien }\end{array}$ & neurologisches Defizit \\
\hline $\begin{array}{l}\text { beitragende } \\
\text { Faktoren }\end{array}$ & $\begin{array}{l}\text { " physisch-biomechanische Einflüsse } \\
\text { " genetische Disposition } \\
\text { - Umweltfaktoren } \\
\text { " Patienten-Verhalten } \\
\text { " psychosoziale Faktoren }\end{array}$ & $\begin{array}{l}\text { - Heben und Tragen schwerer Lasten am Arbeitsplatz } \\
\text { - Besorgnis um die Problematik }\end{array}$ \\
\hline $\begin{array}{l}\text { Therapie und } \\
\text { Management }\end{array}$ & $\begin{array}{l}\text { - Behandlungsplanung } \\
\text { - spezifische gemeinsame Zielsetzung }\end{array}$ & $\begin{array}{l}\text { - Edukation } \\
\text { - schmerzreduzierende und funktionssteigernde } \\
\text { Maßnahmen für den Rücken } \\
\text { - Desensibilisierung des neuralen Systems } \\
\text { - Kräftigung der Beinmuskulatur }\end{array}$ \\
\hline Prognose & $\begin{array}{l}\text { zeitlicher Rahmen für eine realistische Zielsetzung und Veränderung } \\
\text { der Problematik }\end{array}$ & $\begin{array}{l}\text { grundsätzlich „gute“ Prognose: zeitlich } 4 \text { bis } 8 \text { Monate } \\
\text { bis zur Verbesserung oder vollständigen Wiederher- } \\
\text { stellung }\end{array}$ \\
\hline
\end{tabular}

\section{Hypothesenbildung}

Im Anschluss an die Befunderhebung sollte jeder Patient über seine Problematik informiert werden, um dann mit ihm das Ziel der Behandlung gemeinsam festzulegen. Von entscheidender Bedeutung für die meisten Patienten sind v. a. die Fragen, welche Pathologie bei ihnen vorliegt, wieviel Zeit sie für die Rekonvaleszenz brauchen, was der
Therapeut und auch sie selbst zur Genesung beitragen können und wie sie sich zukünftig verhalten sollten, um ein erneutes Trauma zu vermeiden.

Um diese Fragen im vorliegenden Fall zu beantworten, boten die Hypothesenkategorien aus dem Clinical-Reasoning-Prozess eine hilfreiche Unterstützung [1 - 3] ( Tab. 1). 
Kommunikation Im Anschluss erörterte der Therapeut seiner Patientin die möglichen Ursachen für ihre Schmerzen und Bewegungseinschränkungen sowie geeignete Therapieoptionen. So seien die Rückenschmerzen sowie die Beschwerden im Bein die Folge einer Überlastung und Gewebeschädigung der Lendenwirbelsäule. Die Schwäche sowie das „komische Gefühl“ im Bein ließen sich wahrscheinlich auf die Irritation einer Nervenwurzel zurückführen. Am Ende wurde die Patientin über aussichtsreiche Möglichkeiten informiert, ihre Beschwerden durch therapeutische Interventionen und ein Übungsprogramm positiv zu beeinflussen.

\section{Therapieziele}

Die Behandlungsziele formulierte der Therapeut gemeinsam mit Sabine F. anhand des SMART-Prinzips [5]. Gemäß diesem sollten die Ziele spezifisch, messbar, angemessen, realisierbar sowie terminierbar sein.

Für die kommenden drei Wochen wurden folgende Ziele vereinbart:

- Bewegungsausmaß der LWS um 50\% steigern

- Belastbarkeit der LWS auf 1 Stunde Sitzen verbessern

- Linderung der Beinschmerzen auf 1-2/10 NRS

- Ausmaß des SLR rechts auf $50^{\circ}$ erhöhen

- Arbeitsfähigkeit von 4 Stunden/Tag erreichen

- ausreichendes Wissen zum Rückenschmerz

- adäquater Umgang mit der momentanen Situation

\section{Therapieverlauf}

Um die LWS-Beweglichkeit zu steigern und den Beinschmerz zu lindern, wurden in den ersten drei Behandlungseinheiten sowohl aktive, als auch passive Maßnahmen angewandt. Gleichzeitig konnten dosierte aktive Strategien erarbeitet werden, welche der 32-Jährigen den Umgang mit ihrer weiterhin von den Schmerzen dominierten Situation erleichtern sollten. Hierdurch verbesserte sich die Symptomatik in Rücken und Bein, und die Patientin konnte ihre Medikation mit NSAR zeitweise reduzieren.

\section{Edukation}

Anschließend wurde Sabine F. mittels verschiedener Denk- und Erklärungsmodelle aufgeklärt, wieviel sie sich bewegen und belasten dürfe und auch warum der Nerv momentan so überempfindlich reagiere. Bei der Darlegung der Gewebe- und Schmerzmechanismen hielt der Therapeut stets Rücksprache mit seiner Patientin, ob diese die ihr vermittelten Modelle und Informationen verstehen und nachvollziehen konnte.

\section{Pathobiologische Mechanismen}

Gewebemechanismen Bei der 32-jährigen Lageristin führte die Überlastung der unteren LWS beim Heben des schweren Pakets zur Gewebeläsion. Die daraus resultierende Entzündungsphase könnte die Ursache für die Radikulopathie sein. Obwohl sich die Wundheilung bereits kurz nach der Proliferationsphase befand, imponierten in der klinischen Präsentation weiterhin Zeichen entzündlicher Komponenten. Dementsprechend sollte das Gewebe zur strukturellen Ausrichtung im Kollagenbereich belastet werden, und die Therapie sollte bis zu einem spürbaren Gewebswiderstand erfolgen, aber nicht schmerzverstärkend sein [6].

Schmerzmechanismen Des Weiteren informierte der Therapeut über die Nervensensibilisierung und Funktionsstörung. Er erklärte der Patientin, dass ihre Reizschwellensenkung durch eine erhöhte Erregbarkeit und Vermehrung von Ionenkanälen im Nerv als mögliche Ursachen der Beinschmerzen wahrscheinlich seien. Dabei könnte eine Veränderung der Nervenleitgeschwindigkeit das Kraft- und Sensibilitätsdefizit erklären.

Elementar für die Patientin war, dass passive Therapiemaßnahmen oder aktive Übungen niemals Schmerzen verursachen sollten, da es dadurch zur weiteren Sensibilisierung und Verschlechterung ihres Zustands kommen würde [4, $7-10]$.

\section{Angst als Barriere}

Auf den ersten Blick schien es sich im vorliegenden Fall um eine alltägliche Problematik zu handeln. Allerdings verbesserte sich weder die Flexion des Rückens nach den ersten Therapieeinheiten, noch konnte mit der Patientin der Widereinstieg in ihre berufliche Tätigkeit angemessen besprochen werden.

Kern des Problems Nach weiterer Abklärung psychosozialer Risikofaktoren bzw. ,Yellow Flags‘ sowie der Rückfrage des Therapeuten äußerte die Patientin nun explizit ihre Angst vor einer Schmerzverstärkung durch Aktivität sowie die Befürchtung, ihren Rücken durch die Arbeit als Lageristin noch weiter zu schädigen [11].

\section{Anpassung der Behandlungsstrategie}

Aufgrund dieser neuartigen Situation wurde die Behandlungsstrategie modifiziert. Als weitere Ziele der Therapie wurden nun formuliert:

- weniger Angst vor Schmerz und Schädigung

- Wissen zum Thema Schmerzphysiologie

Diese Themen wurden unter dem Aspekt des ,Graded Exposure‘ und zugehöriger Denkmodelle adressiert [3,9]. 


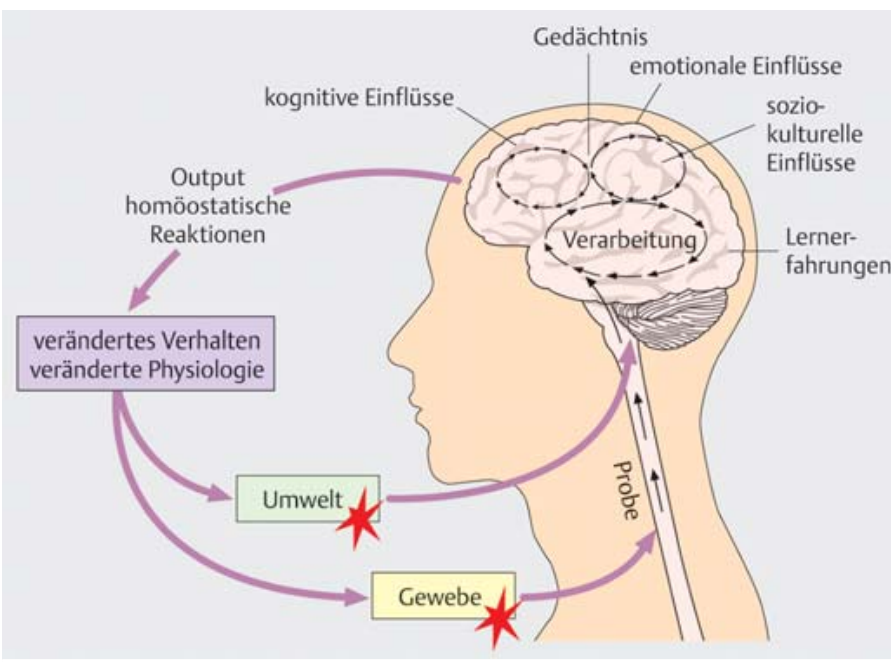

- Abb. 2 Das Mature Organism Model [4, 12]. Quelle: Stam H. Nozizeptive Schmerzmechanismen. In: Westerhuis P, Wiesner R (Hrsg). Klinische Muster in der Manuellen Therapie. Stuttgart: Thieme; 2011.

\section{,Mature Organism Model‘}

Für die Erklärung der Bedeutung von Schmerzmechanismen und beitragender Faktoren wurde das ,Mature Organism Model' (MOM) von Louis Gifford gewählt [4] ( Abb. 2).

Das MOM betrachtet eine Verletzung als "gewebespezifischen Faktor“. Die Schwere der Verletzung bestimmt nicht exklusiv die Intensität der Schmerzen, sondern steht im Wechselspiel mit der Lebens- und Schmerzgeschichte des Betroffenen. Anschaulich wird dies durch den „individuellen Schmerzkuchen“ jedes Patienten [3]. Dieser entsteht durch das Zusammenwirken seiner Zutaten und ist das Ergebnis eines emergenten Prozesses.

Beitragende Faktoren Giffords Denkmodell ist hervorragend geeignet, um einem Patienten ein biopsychosoziales Verständnis für seine individuelle Schmerzsituation zu vermitteln und ihm die entsprechenden Zusammenhänge zu erklären. Als wichtige zum Schmerzgeschehen beitragende Faktoren konnten nun die beiden Aspekte „Angstvermeidung“ und „körperliche Forderung am Arbeitsplatz“ identifiziert werden.

\section{Schmerzmodulation auf Rückenmarkebene}

Um der Patientin eine weitere Möglichkeit der Schmerzmodulation begreiflich zu machen, wurden ihr anschauliche Illustrationen aus dem Buch „Schmerzen verstehen“ von David Butler und Lorimer Moseley vorgestellt [8]. Anhand derer werden u.a. die neuronale Verschaltung im Rückenmark sowie sich daraus ergebenden Einflüsse auf die Schmerzwahrnehmung auch für medizinische Laien verständlich.

Schmerzregulation Im Zentrum der Edukation stand die nun vermehrt apparent werdende „Schmerzregulation nach oben" durch Ängste und das vermehrte Fokussieren auf das Problem. Angestrebt wurde dagegen eine "Schmerzregulation nach unten“ über Entspannungstechniken, die Steigerung der allgemeinen Fitness, das Gefühl der Sicherheit sowie positive Gedanken. Um die im therapeutischen Setting vorgestellte Schmerzmodulation auf Rückenmarksebene und deren Hintergründe zu verinnerlichen, erhielt die Patientin das Buch zur Heimlektüre. Im Folgetermin konnten diesbezüglich noch offene Fragen geklärt werden.

\section{Formen der Angst}

Die Kommunikation zum Thema Angst ist für jeden Therapeuten eine Herausforderung. Nicht selten interpretiert ein Patient seine Angst als Schwäche und vermeidet, diese in der Behandlung zu thematisieren. Als Befragungs- und Screening-Möglichkeiten zur Evaluierung von Ängsten haben sich die ,Tampa Scale of Kinesiophobia“ (TSK) und der ,Fear-Avoidance Beliefs Questionnaire“ (FABQ) bewährt.

Im vorliegenden Fall traten erst im Verlauf der Therapie zwei unterschiedliche Arten von Angst zutage - zum einen die Angst vor einer Schmerzverstärkung bei Aktivität, zum anderen die Angst vor einer weiteren Schädigung von Rücken und Nerv durch Bewegung und Belastung. Diese Ängste der 32-jährigen Patientin wurden im Folgenden differenziert betrachtet und behandelt.

\section{Angst vor Schmerzverstärkung}

Um die Angst vor einer Schmerzverstärkung zu thematisieren, eignen sich das angeführte ,Mature Organism Model' sowie die Edukation zur Schmerzphysiologie mittels „Schmerzen verstehen“ [8]. Zur Intensivierung der Edukation eignen sich auch einige Videoclips im Internet. 


\section{VIDEOS ZUR SCHMERZEDUKATION}

Folgende YouTube-Videos eignen sich sehr gut zur

Schmerzedukation [13]:

- Brainman:

- „Was ist Schmerz und wie kann ich ihn lindern?"

- „Schmerzen verstehen: Brainman wird aktiv“

- „Schmerzen verstehen: Brainman stoppt seine Opiate“

- Deutsches Kinderschmerzzentrum Datteln:

- „Den Schmerz verstehen und was zu tun ist in 10 Minuten"

- Tamar Pincus:

- „Schmerz und ich“

- pt-Fachvideo:

- „Protectometer“

\section{Angst vor Neuverletzung}

Um ihrer Angst vor einer erneuten Schädigung entgegenzuwirken, sollte die Patientin mit therapeutischer Unterstützung lernen, ihrer Wirbelsäule und deren Belastbarkeit wieder mehr zu vertrauen. Dieser Teil der Edukation gestaltete sich bei Sabine F. allerdings hochproblematisch, da sie sehr stark auf ihre bildgebenden Befunde fokussiert war: „Ja, aber ich habe doch einen Bandscheibenvorfall“.

Wundheilungsphasen Hilfreich für die Patientin waren Informationen über die Wundheilungsphasen. Konkret wurde der 32-Jährigen aufgezeigt, dass die neu gebildeten kollagenen Fasern sich nur durch entsprechende Bewegungen und Belastung adäquat im Zellverband ausrichten können, um eine gute LWS-Stabilität zu gewährleisten. In diesem Sinne informierte der Therapeut, wie Belastung und Belastbarkeit miteinander korrelieren. Gemäß dem Prinzip „Use it or lose it“ werden Gewebsstrukturen bei Gebrauch kräftiger und stabiler.

\section{Graded Exposure}

Das Angst-Vermeidungsverhalten ist ein sehr häufiges Problem bei Rückenschmerzpatienten [14]. Die Veränderung dieses maladaptiven Verhaltensmusters ist jedoch wesentlich für den Erfolg einer Therapie. ,Graded Exposure' bietet eine gute Möglichkeit, dieses Ziel mittels einer abgestuften Expositionstherapie zu erreichen [9, 15].
KERNASPEKTE DES GRADED EXPOSURE

- Erstellung einer Angsthierarchie: Erfassen der problematischen Aktivitäten/Situationen

- Aufklärung/Edukation

- Beginn mit einer wenig angstassoziierten Aktivität

- Reflexion nach durchgeführter Aktivität

- Steigerung mit der nächsten Aktivität auf der Angsthierarchie bei Reduktion oder Inapparenz der Ängste

- bewusste Konfrontation zur Steigerung des Aktivitätsniveaus

Angsthierarchie Als am meisten mit Angst behaftete Aktivität gab die Patientin an, etwas Schweres aufzuheben und zu tragen. Als zweite Aktivität in ihrer Angsthierarchie folgte die Flexion des Rückens im Stand. Etwas besser beurteilte sie das Sockenanziehen im Sitzen.

Steigerung Als initiale Aktivität diente das wiederholte Beugen des Rückens im Sitzen, weil dieses bei der Patientin weniger mit Angst assoziiert war. Für einen nachhaltig positiven Effekt sollte Frau F. diese Übung auch zuhause regelmäßig ausführen. Ihre ängstlichen Bedenken verringerten sich binnen kurzer Zeit, weil sich die Symptomatik durch das Bewegen nicht verschlechterte. Im Anschluss führte die Patientin dann andere Aktivitäten ihrer individuellen Angsthierarchie aus.

\section{Retrospektive}

Die Behandlung von Sabine F. dauerte acht Wochen. Der Verlauf gestaltete sich weitaus schwieriger als erwartet, was schließlich die Rehabilitation der 32-jährigen verzögerte. Mehr als die Hälfte aller Therapieeinheiten wurden für die Edukation verwendet. Bis zum jetzigen Zeitpunkt hat die Lageristin ihre berufliche Tätigkeit nicht wiederaufgenommen.

Persistierende Probleme Bei einer retrospektiven Analyse offenbaren sich diverse Barrieren auch für die zukünftige Therapie. So stellen die persönliche Einstellung sowie die kinesiophoben Gedanken der Patientin weiterhin beträchtliche beitragende und limitierende Faktoren ihrer Regeneration und Wiederaufnahme der beruflichen Tätigkeit und sportlichen Aktivität dar. Nach wie vor sind die MRT-Befunde sowie die Diagnose „Bandscheibenvorfall“ für sie ein gravierendes Problem. Demgemäß kommunizierte sie im späteren Therapieverlauf unablässig und formelhaft, dass sie „aber diesen Bandscheibenvorfall und deswegen Schmerzen“ habe und sich "nicht bewegen oder arbeiten“ könne. Diese immer wiederkehrenden Aussagen standen für den Therapeuten in deutlicher Analogie zu den Items der TSK und des FABQ. 
Erkenntnisse Im Rückblick wäre es sicherlich besser gewesen, bereits früher auf die Ängste und dysfunktionalen Kognitionen der Patientin einzugehen und diese explizit via TSK und FABQ zu erfassen. Dann allerdings wäre die intensive Edukation zu kurz gekommen. Der geschilderte Behandlungsverlauf zeigt die Schwierigkeit, die Therapie in der Frühphase der Rehabilitation mit einer Angstreduktion durch Graded Exposure zu kombinieren. Offen bleibt die Frage, ob mit mehr Zeit sowie einer höheren Therapiedichte zu Beginn ein besseres Behandlungsergebnis hätte erzielt werden können.

Die anfänglich gute Prognose wurde revidiert und die Therapie im Verlauf angepasst. Zum gegenwärtigen Zeitpunkt ist das Therapieergebnis sowohl für den Patienten als auch für den Behandler nicht zufriedenstellend.

\section{Fazit}

In der Physiotherapie gibt es keinen Königsweg und keine Patentrezepte. Die Individualität jedes Menschen bedarf einer spezifischen Kommunikation und Therapieplanung je nach klinischer Präsentation, Erfahrung des Therapeuten und Verständnis des Patienten. Selbst aktuelle wissenschaftliche Erkenntnisse können hier nur bedingt helfen.

Ein Kommunikationsstil auf Augenhöhe erscheint auf den ersten Blick zwar einfach, bedarf immer aber einer Anpassung an den Patienten und dessen individueller Problematik. Sprache, Verständnis, Hintergrundwissen, unterschiedliche Erfahrungen und auch kulturelle Hintergründe haben einen Einfluss auf das Assessment und die Therapie. Oft gibt es aber Ursachen für das als „ungewöhnlich“, „schwierig“ oder gar „faul“ anmutende Verhalten von Patienten, wie zum Beispiel Ängste. Diese gilt es, frühzeitig in der Therapie zu entlarven und konkret zu adressieren.

Das aktuell bestmöglichste Procedere bietet scheinbar ein fundiertes Clinical Reasoning kombiniert mit der Anwendung des ,Mature Organism Model'. Anhand des Fallbeispiels wird deutlich, wie stark die Gedankenspiele und Bewertungen eines Patienten dessen Genesung beeinflussen. Dies unterstreicht, wie wichtig ein gutes Screening dieser Faktoren und eine entsprechend angepasste Edukation sind.

\section{Autorinnen/Autoren}

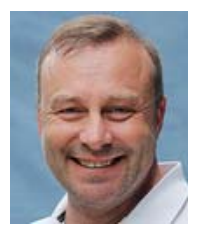

Ralf Schesser ist Physiotherapeut an der Fachklinik Enzensberg und als Dozent in der Weiterbildung „Spezielle Schmerzphysiotherapie“ der Deutschen Schmerzgesellschaft e.V., der OMPT-Ausbildung sowie an Hochschulen und Universitäten tätig. Er ist Mitautor des Curriculums Spezielle Schmerzphysiotherapie und Mitherausgeber der Zeitschrift „Der Schmerzpatient".

\section{Korrespondenzadresse}

\section{Ralf Schesser}

Interdisziplinäres Schmerzzentrum

Fachklinik Enzensberg

Höhenstr. 56

87629 Hopfen a. S.

ralfschesser@hotmail.com

\section{Literatur}

[1] Jones M. Clinical reasoning: the foundation of clinical practice. Part 1. Aust J Physiother 1997; 43: 167-170

[2] Jones MA, Rivett D. Clinical Reasoning fo Manual Therapists. Oxford: Butterworth Heinemann; 2003

[3] Luomajoki H, Schesser R. Schmerzmechanismen und Clinical Reasoning. Schmerzpatient 2018; 1 (01): 7-18. doi:10.1055/ s-0043-122097

[4] Gifford L. Schmerzphysiologie. In: van den Berg F, Hrsg. Angewandte Physiologie 2. Stuttgart: Thieme; 2005: 467-518

[5] McGrath J, Kischka UC. Interdisziplinäre Teamarbeit und Zielsetzung in der Rehabilitation. In: Frommelt P, Lösslein $\mathrm{H}$, Hrsg. NeuroRehabilitation: Ein Praxisbuch für interdisziplinäre Teams. Heidelberg: Springer; 2010: 107-114

[6] van den Berg F. Angewandte Physiologie. Band 1: Das Bindegewebe des Bewegungsapparates verstehen und beeinflussen. Stuttgart: Thieme; 2016

[7] Butler D. The Sensitive Nervous System. Adelaide: NOlgroup Publications; 2000

[8] Butler D, Moseley GL. Schmerzen verstehen. Heidelberg: Springer Medizin Verlag; 2016

[9] Gifford L. Aches and Pain. Book 1-3. Cornwall: WordPress; 2014

[10] Moseley GL, Butler D. Explain Pain Supercharged. Adelaide: NOIpress; 2017

[11] Waddell G. The Back Pain Revolution. Edinburgh: Churchill Livingstone; 2004

[12] Stam H. Nozizeptive Schmerzmechanismen. In: Westerhuis P, Wiesner R, Hrsg. Klinische Muster in der Manuellen Therapie. Stuttgart: Thieme; 2011

[13] Egan Moog M. Ein Plädoyer für chronische Schmerzpatienten. Der Schmerzpatient 2018; 3 (01): 112-122. doi:10.1055/a0579-2101

[14] Vlaeyen JW, Crombez G, Linton S. The fear-avoidance model of pain. PAIN 2016; 157 (8): 1588-1589. doi:10.1097/j.pain. 0000000000000574

[15] Macedo LG, Smeets R], Maher CG et al. Graded Activity and Graded Exposure for Persistant Nonspecific Low Back Pain: A Systematic Review. Physical Therapy 2010; 90 (6): 860-879. doi:10.2522/ptj.20090303.

\section{Bibliografie}

DOI https://doi.org/10.1055/a-0969-4159

Der Schmerzpatient 2020; 3: 40-46

(c) Georg Thieme Verlag KG Stuttgart · New York ISSN 2512-6210 